\title{
OPERATOR REPRESENTATION THEOREMS
}

\author{
RICHARD H. HERMAN ${ }^{1}$
}

We represent certain types of bounded linear operators from a Banach space into a space $\mathrm{BC}(S)$ where $S$ is an arbitrary topological space and $\mathrm{BC}(S)$ is the space of bounded continuous scalar-valued functions on $S$ with the sup norm. A comprehensive treatment of work to date may be found in [1] and [7]. We represent the completely continuous (not to be confused with compact) operator, which has not been considered previously. This yields a sufficient condition for $T: X \rightarrow \mathrm{BC}(S)$ to be strictly singular.

Definitions. (a) $A$ set $U \subset X$ is said to be absolutely convex if it is convex and $\lambda U \subset U$ for $|\lambda| \leqq 1$. (b) We denote the smallest closed absolutely convex set containing $W$ by $\operatorname{aco}(W)$. Let $U \subset X$. By $U_{0}$ (the polar of $U$ ) we shall mean $\left\{x^{*}|| x^{*}(u) \mid \leqq 1\right.$ for all $\left.u \in U\right\}$. (c) If $U \subset X^{*}$ we define $U_{0}=\{x|| u(x) \mid \leqq 1$ for all $u \in U\}$. We write $U^{0}{ }_{0}$ for $\left(U^{0}\right)_{0}$.

The Mackey topology on $X^{*}$ is that topology generated by the polars of all absolutely convex, weakly compact sets of $X[6, \mathrm{p} .173]$.

A bounded linear operator $T: X \rightarrow Y$ is said to be completely continuous if it maps weakly convergent sequences into norm convergent sequences. (This is the original definition of Hilbert, which coincides with compactness when $X$ is reflexive.)

Lemma 1. Let $A \subset X^{*}$. If $A$ is Mackey conditionally compact, then given any sequence $\left\{x_{n}\right\} \subset X$ such that $x_{n} \rightarrow x$ in the weak topology, it follows that $x_{n} \rightarrow x$ uniformly on $A$.

Proof. Suppose $\epsilon>0$ and $x_{n} \rightarrow 0$ weakly. Then $\left\{x_{n}, 0\right\}$ is weakly compact. By the Krein-Smulian theorem [1, p. 434], $V=\operatorname{aco}\left\{x_{n}, 0\right\}$ is also weakly compact. By hypothesis there exist $x_{i}^{*}, i=1, \cdots, k$ such that

$$
A \subset \bigcup_{1}^{k}\left(x_{i}^{*}+\frac{\epsilon}{4} V^{0}\right)
$$

Picking $N$ so large that $\left|x_{i}^{*}\left(x_{n}\right)\right|<\epsilon / 2$ for $n>N$ and $i=1, \cdots, k$ the result follows.

The lemma above is a corollary in $[4$, p. 134].

The following lemma is due to Elton Lacey.

Received by the editors January 10, 1967.

1 This paper is taken from Chapter III of the author's doctoral dissertation and was done while the author was a NDEA Fellow at the University of Maryland. 
Lemma 2. The following are equivalent for $T: X \rightarrow Y$ :

(a) $T$ is completely continuous.

(b) $T^{*}$ is continuous as a map from the bounded sets of $Y^{*}$ with the weak* topology to $X^{*}$ with its Mackey topology.

(c) If $B \subset X^{*}$ is weak* compact then $T^{*}(B)$ is Mackey compact.

Proof. (a) implies (b). Let $V$ be an absolutely convex weakly compact set in $X$. It suffices to show that there exists a finite set $\left\{y_{1}, \cdots, y_{n}\right\} \subset Y$ such that $T^{*}(W) \subset V^{0}$ where $W=S\left(Y^{*}\right)$ $\bigcap a\left(\left\{y_{1}, \cdots, y_{n}\right\}\right)^{0}$ and $S\left(Y^{*}\right)$ is the unit ball in $Y^{*}$. Since $T$ is completely continuous, $T V$ is compact. If $\left\{y_{1}, \cdots, y_{n}\right\}$ is an $\epsilon$-net for $T V$ and $a=1-\epsilon$, then the corresponding $W$ is seen to work.

(b) implies (c) is clear.

(c) implies (a) follows from $[4$, p. 132].

Henceforth $X$ and $Y$ denote Banach spaces and $T$ a bounded linear operator. When we refer to a $\mathrm{BC}(S)$ space, it is as mentioned in the introduction.

Theorem 3. Let $T: X \rightarrow \mathrm{BC}(S) . T x(s)=p(s) x$ where $p: S \rightarrow X^{*}$ is weak* continuous and $p(S)$ is bounded. $T$ is completely continuous if and only if $p(S)$ is Mackey conditionally compact.

Proof. The representation of any bounded map by such a $p(\cdot)$ is shown in [7].

Now suppose $p(S)$ is Mackey conditionally compact. We show that $T$ maps weak Cauchy sequences into norm Cauchy sequences. Let $\left\{x_{n}\right\} \subset X$ be weak Cauchy and let $\left\{n_{j}\right\}$ be any increasing sequence of integers. Then $\left\{x_{n_{j-1}}-x_{n_{j}}\right\} \rightarrow 0$ weakly. By Lemma 1, given $\epsilon>0$, there exists $N(\epsilon)$ such that for $n_{j}>N$

$$
\sup _{s \in S}\left|p(s)\left(x_{n_{j-1}}-x_{n_{j}}\right)\right|<\epsilon=\sup _{s \in S}\left|T\left(x_{n_{j-1}}-x_{n_{j}}\right)(s)\right|,
$$

i.e., for every $\left\{n_{j}\right\},\left\{T\left(x_{n_{j-1}}-x_{n_{j}}\right)\right\} \rightarrow 0$ in norm. Thus $\left\{T x_{n}\right\}$ is Cauchy in norm.

Conversely, suppose $T$ is completely continuous. By Lemma 2, $T^{*}$ takes weak* compact sets in to Mackey compact sets. We have $p=T^{*} \circ \pi$ where $\pi: S \rightarrow \mathrm{BC}(S) *$ is defined by $\pi(s) f=f(s) . \pi(S)$ is contained in the unit ball of $\mathrm{BC}(S) *$ which is weak* compact; hence, $p(S)=T^{*} \circ \pi(S)$ is Mackey conditionally compact.

Corollary 4. If $S$ is a compact topological space, then $T: X \rightarrow C(S)$ is completely continuous if and only if $p$ is continuous as a map from $S$ with its own topology to $X^{*}$ with the Mackey topology.

Proof. If $p$ is continuous in the Mackey topology, then since $S$ is 
compact, $p(S)$ is Mackey compact. Thus, $T$ is a fortiori completely continuous.

If $T$ is completely continuous, using Lemma 2 we see that $T^{*}$ is a weak*-Mackey continuous map on bounded sets. Thus, $T^{*}$ is weak*Mackey continuous on $\pi(S)$. But $\pi$ is a continuous map between $S$ with its own topology and $\pi(S)$ with the weak* topology. Therefore, $p=T^{*} \circ \pi$ is continuous in the Mackey topology.

Definition. $T: X \rightarrow Y$ is said to be strictly singular if whenever $T$ has a bounded inverse on a subspace $M \subset X$, then $M$ is finite dimensional $[2$, p. 76].

Corollary 5. Let $T: X \rightarrow \mathrm{BC}(S)$; then, if $p(S)$ is weakly conditionally compact and Mackey conditionally compact, $T$ is strictly singular.

Proof. The weak conditional compactness guarantees [7] that $T$ is weakly compact. In addition, by Theorem $3, T$ is completely continuous. Any such operator is strictly singular, for suppose $T$ has a bounded inverse on $M . M$ is then reflexive since $T$ is weakly compact. $S(M)$ is then weakly compact. By the complete continuity of $T$, $T S(M)$ is compact and, since $T$ has a bounded inverse, $S(M)$ is compact. Thus $M$ is finite dimensional.

We do not obtain all of the strictly singular operators in this manner, as the following example shows.

Let $T: l^{1} \rightarrow c_{0}$ be given by $T\left(\left(\alpha_{i}\right)\right)=\left(\sum_{j=i}^{\infty} \alpha_{j}\right)$. Taking the standard basis $\left\{e_{n}\right\}$ in $l^{1}$ we see that $T$ is not weakly compact; however, $T$ is strictly singular [3]. Let $A$ be the isomorphism between $c_{0}$ and $c$. Then $A \circ T: l^{1} \rightarrow c$ is not weakly compact but is a strictly singular map into a $C(S)$ space ( $S$ is the one-point compactification of the integers). But $A \circ T$ is clearly completely continuous since norm and weak sequential convergence are the same in $l^{1}[1$, p. 296].

If we restrict $X$, it is possible to weaken the conditions on $p$ and still retain strict singularity. A Banach space $X$ is said to be infrareflexive if every infinite dimensional closed subspace $M$ contains an infinite dimensional reflexive subspace. It is known that this class of spaces contains properly the class of quasi-reflexive spaces. For this and related results, see $[5]$.

Corollary 6. Let $T: X \rightarrow C(S)$ where $X$ is infrareflexive and $S$ is a compact topological space. If $p$ is Mackey continuous, then $T$ is strictly singular.

Proof. It suffices to show [2, p. 84] that for any infinite dimensional subspace $M$, there exists $N \subset M, N$ infinite dimensional such 
that $T_{N}$ is compact. Let $M$ be one such subspace. By hypothesis, $M$ contains $N$ infinite dimensional and reflexive. Thus $S(N)$ is weakly compact. Since $p$ is Mackey continuous given $\epsilon>0$ and $s_{\alpha}$, a net in $S$ converging to $s$, it follows that there exists $\alpha_{0}$ such that for $\alpha>\alpha_{0}$

$$
\left|\left(p\left(s_{\alpha}\right)-p(s)\right) x\right|<\epsilon
$$

for $x \in S(N)$. That is, $p_{N}$ is norm continuous and, by [7, Corollary 8], $T_{N}$ is compact.

We now apply Lemma 1 to the following result from $[1$, p. 503].

TheOREM. Let $(S, \Sigma, \mu)$ be a $\sigma$-finite measure space and $T: L_{1}(S, \Sigma, \mu)$ $\rightarrow X^{*}, X$ separable. Then there exists a $\mu$-essentially unique function $x^{*}(\cdot)$ on $S$ to $X^{*}$ such that for each $x, x^{*}(\cdot) x \in L_{\infty}(S, \Sigma, \mu)$ and

$$
\begin{aligned}
(T f) x & =\int_{S} x^{*}(s) x f(s) \mu(d s), \quad f \in L_{1}(S, \Sigma, \mu), \\
\|T\| & =\underset{s \in S}{\operatorname{ess} \sup }\left\|x^{*}(s)\right\| .
\end{aligned}
$$

If we let $V=\left\{x^{*}(\cdot) x \mid\|x\|<1\right\}$, then

CoROLlaRy 7. With $T$ as above, $T$ is completely continuous if and only if $V$ is Mackey conditionally compact.

Proof. Suppose $V$ is Mackey conditionally compact. To show $T$ is completely continuous, it suffices to show that if $f_{n} \rightarrow 0$ weakly, then $T f_{n} \rightarrow 0$ in norm (see the proof of Theorem 3). Let $\left\{f_{n}\right\}$ be one such sequence. By the hypothesis and Lemma $1, f_{n} \rightarrow 0$ uniformly on $V$, i.e., given $\epsilon>0$ there exists $N(\epsilon)$ such that for $n>N$

$$
\left|\left(T f_{n}\right) x\right|=\left|\int_{S} x^{*}(s) x f_{n}(s) \mu(d s)\right|<\epsilon \text { for } x,\|x\| \leqq 1 .
$$

Therefore, $T f_{n} \rightarrow 0$ in norm.

Conversely, we note that $V=T^{*} \cdot J S(X)$ for $(T f) x=J x(T f)$ $=T^{*}(J x) f=\int x^{*}(s) x f(s) \mu(d s)=x^{*}(\cdot) x(f)$. Since $T$ is completely continuous, by Lemma 2 we have that $V$ is Mackey conditionally compact.

The author thanks Seymour Goldberg for encouragement and advice.

\section{BiBLIOGRAPHY} 1958.

1. N. Dunford and J. Schwartz, Linear operators, Part I, Interscience, New York, 
2. S. Goldberg, Unbounded linear operators, McGraw-Hill, New York, 1966.

3. S. Goldberg and E. O. Thorp, On some open questions concerning strictly singular operators, Proc. Amer. Math. Soc. 14 (1963), 334-336.

4. A. Grothendieck, Sur les applications lineaires faiblement compacts d'espaces du type $C(K)$, Canad. J. Math. 5 (1953), 129-173.

5. R. Herman and J. Whitley, An example concerning reflexivity, Studia Math. 28 (1967), 289-294.

6. J. L. Kelley and I. Namioka, Linear topological spaces, Van Nostrand, Princeton, N. J., 1963.

7. E. O. Thorp and R. J. Whitley, Operator representation theorems, Illinois J. Math. 9 (1965), 595-601.

UNIVERSITY OF MARYLAND 